\title{
Blues clues
}

\section{Sara Ashraf, Ali Aamar}

Department of Internal Medicine, Yale-New Haven Hospital Ring Gold Standard Institution, New Haven, USA

Correspondence to: Sara Ashraf. Department of Internal Medicine, Yale-New Haven Hospital Ring Gold Standard Institution, 20 York Street, New Haven, Connecticut 06510-3202, USA. Email: sara.ashraf@yale.edu.

Received: 29 April 2017; Accepted: 25 May 2017; Published: 11 July 2017.

doi: 10.21037/sci.2017.06.06

View this article at: http://dx.doi.org/10.21037/sci.2017.06.06

A 70-year-old female with recently diagnosed extramedullary acute myeloid leukemia (AML) presented with severe shortness of breath and chest pain found to have

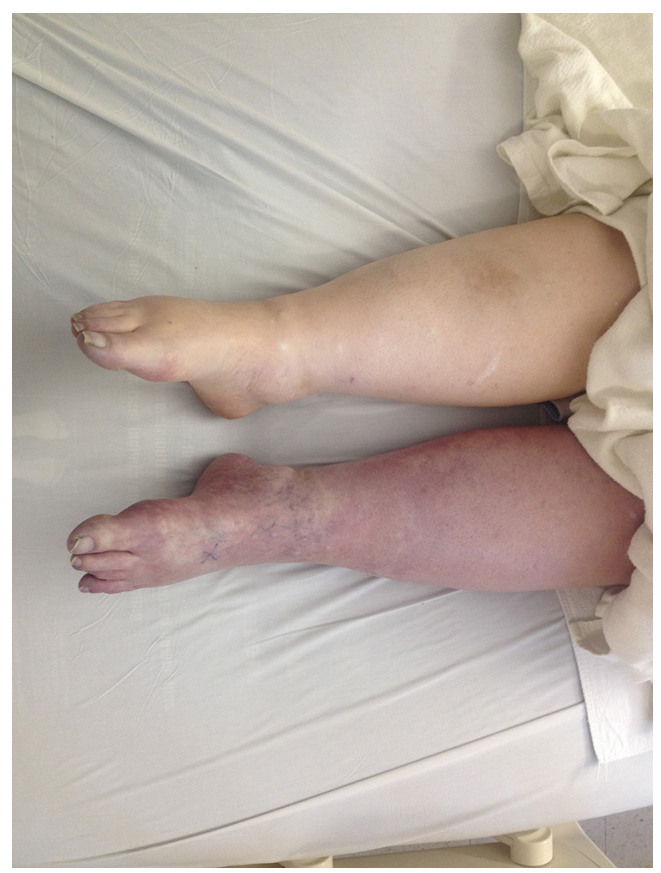

Figure 1 Patient with blue edema of the left lower extremity secondary to extensive deep venous thrombosis. a massive pulmonary embolism. On physical examination, the patient had acute phlegmasia cerulea dolens (painful blue edema) of her left lower extremity (Figures 1 and 2)

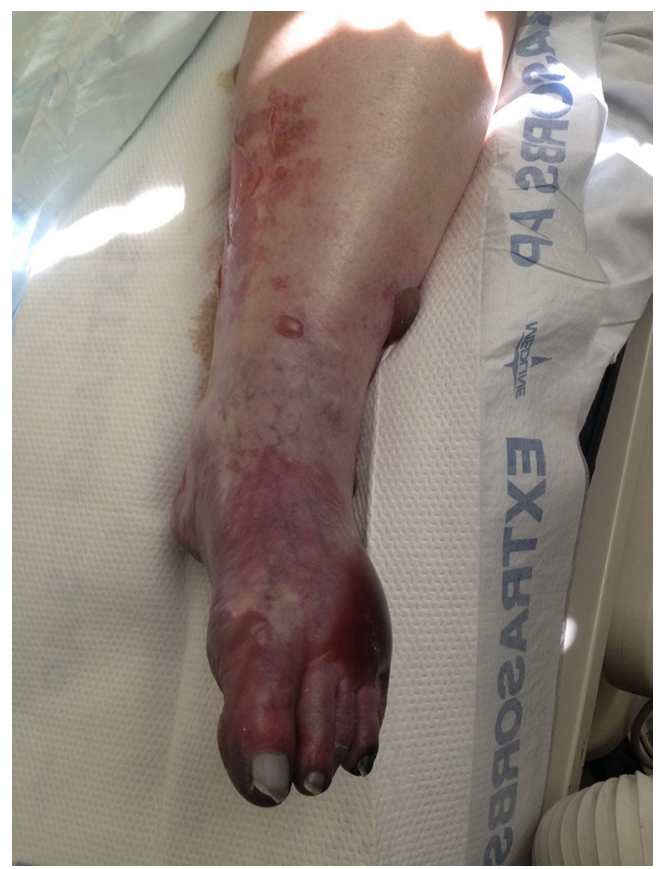

Figure 2 Patient with blue edema of the left lower extremity secondary to extensive deep venous thrombosis. 


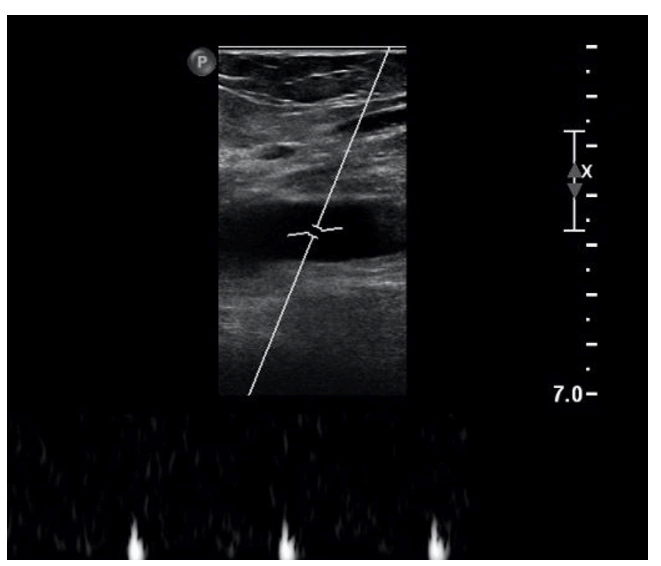

Figure 3 The patient with extensive occlusive deep venous thrombosis of right common femoral vein on ultrasound imaging.

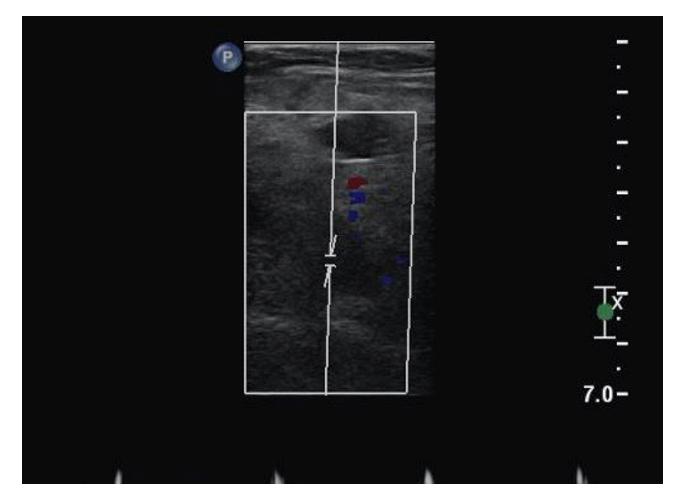

Figure 4 The patient had occlusive deep venous thrombosis of left external iliac vein on ultrasound imaging.

doi: $10.21037 /$ sci.2017.06.06

Cite this article as: Ashraf S, Aamar A. Blues clues. Stem Cell Investig 2017;4:61. secondary to extensive bilateral deep vein thrombosis (DVT) up to the proximal common iliac veins. Occlusive deep vein thrombosis of right common femoral vein (Figure 3) and that of left external iliac vein (Figure 4) are visible on Doppler ultrasound.

Phlegmasia cerulea dolens is an uncommon, severe deep venous thrombosis arising from occlusion of both major and collateral veins of a limb. Patients complain of sudden extreme pain, swelling, edema and bluish/purplish discoloration of the affected extremity. Like in our patient, $50 \%$ are associated with an underlying malignancy and it can lead to massive pulmonary embolism or gangrene.

Unfortunately, the patient did not survive despite immediate anticoagulation.

\section{Acknowledgements}

None.

\section{Footnote}

Conflicts of Interest: The authors have no conflicts of interest to declare.

Informed Consent: The patient consented to all authors the use of photographs and publication of this image report. 